\title{
IRISH CARDIAC SOCIETY
}

\author{
Proceedings of the Annual General Meeting held November 1993.
}

\section{SESSION 1}

ISIS-4: RANDOMISED TRIAL OF ORAL CAPTOPRIL, ISOSORBIDE MONONITRATE AND OF INTRAVENOUS MAGNESIUM IN 58,000 PATIENTS WITH SUSPECTED ACUTE MYOCARDIAL INFARCTION

D. O'Callaghan, J. H. Horgan on behalf of the ISIS-4 Collaborative Group

Beaumont Hospital, Dublin.

58,000 patients were randomised in ISIS-4 from over 1000 hospitals in 30 countries between July 1991 and 31 August 1993. Patients were eligible if they presented within 24 hours of suspected acute myocardial infarction with no clear indications for or contraindications to, the study treatments (although planned use of a few days of intravenous or oral nitrates was permitted). About $40 \%$ were within 6 hours of pain onset, $75 \%$ had ST elevation, $25 \%$ were aged $70+, 15 \%$ had heart failure, and $2 \%$ had systolic blood pressure $<100 \mathrm{mmHg}$

Patients were randomly allocated in a $2 \times 2 \times 2$ factorial design between one month of oral captopril $6.25 \mathrm{mg}$ initial dose, 12.5 mg 2 hours later, $25 \mathrm{mg} \mathrm{10-12}$ hours later and then $50 \mathrm{mg}$ twice daily) versus placebo, one month of oral controlled-release isosorbide mononitrate (Imdur: $30 \mathrm{mg}$ initial dose, $30 \mathrm{mg}$ 10-12 hours later and then $60 \mathrm{mg}$ each morning) versus placebo, and 24 hours of intravenous magnesium sulphate $(8 \mathrm{mmol}$ initial bolus over 15 minutes followed by $72 \mathrm{mmcl}$ ) versus open control. About $75 \%$ received fibrinolytic and almost all antiplatelet therapy.

The main comparisons are to be of 5-week and longer-term mortality amongst all those allocated each active therapy versus all those allocated the corresponding control. Principal subsidiary comparisons involve subdivison by planned nitrate at entry and by the other randomly allocated treatments. Mode of death and major morbidity results will also be considered.

REFERRAL FOR CORONARY ANGIOGRAPHY AFTER AN EXERCISE ECG: DECISION ANALYSIS COMPARED WITH AN INDIVIDUAL CLINICIAN.

\section{J. Kellett, *J. Graham.}

General Hospital, Nenagh, Co. Tipperary.

*West Memorial Regional Hospital, Corner Brook, Newfoundland, Canada.

The decisions by a single cardiologist as to which of 308 consecutive patients to refer for angiography after treadmill testing were compared with their life expectancy gains from bypass surgery predicted by decision analysis. Neither patient age nor gender influenced the decision to perform angiography. The 94 patients sent for angiograms exercised for a significantly shorter time $(\mathrm{p}<0.001)$, had more ST deviation $(\mathrm{p}<0.001)$, more angina $(p<0.002)$ and were more likely to have had a prior myocardial infarction $(p<0.001)$ than the 214 patients not referred. The mean life expectancy gain predicted from bypass surgery was also greater $(p<0.001)$ in those referred $(2.9 \pm 1.7$ QALYs) than in those not referred for angiography $(1.0 \pm 1.7$ QALYs). However, 143 patients not referred were predicted to gain up to 5.7 QALYs from bypass surgery. Consequently the overall predicted life expectancy gain from the cardiologist's intuitive decisions was only $0.1 \pm 2.5$ QALYs per patient. Had the referral decision been solely directed by decision analysis the overall gain per patient would have been 1.9 \pm 1.6 QALYs, and 135 extra patients (229 in total) would have been sent for angiography. Use of decision analysis, therefore might help make referral for angiography more efficient and consistent.

HIGH INCIDENCE OF OESOPHAGEAL ABNORMALITIES DETECTED IN PATIENTS WITH TREATED CORONARY ARTERY DISEASE USING 24HR AMBULATORY MANOMETRY AND $\mathrm{pH}$ STUDIES

B. Deb, M. T. P. Caldwell; P. O'Callaghan, P. J. Byrne, T. P J. Hennessy, P. Crean, M. Walsh, G. Gearty.

Departments of Surgery and Cardiology, St. James's Hospital, Dublin 8.

Persistent chest pain with normal cardiac investigations is not uncommon following treatment of coronary artery disease. Oesophageal problems are often suspected but to date evaluation has proved difficult. Eight patients who had previously undergone successful coronary artery bypass grafting or coronary angioplasty underwent $24 \mathrm{hr}$ ambulatory manometry, $\mathrm{pH}$ and ECC monitoring. Symptoms were correlated with the parameters measured.

Results: Six of 8 patients had pathological acid reflux and one had nutcracker oesophagus. One had a normal study. Seven of the 8 patients reported a total of 63 pain events during the study period. Fifty (79\%) of these events were reflux related and all 63 $(100 \%)$ had an associated motility abnormality. The patient who had a normal study had 6 episodes of pain, all of which were associated with acid reflux events even though his overall profile was within normal limits.

These data suggest that oesophageal events are a frequent cause of pain in patients with IHD even when the $24 \mathrm{hr}$ profile is considered within normal limits. Twenty four hour studies increase the diagnostic yield.

BLMCC EAST OF THE RIVER. MOBILE CORONARY CARE, MARKET FORCES, MINISTERS AND THE MEDIA

D. McC. Boyle, J. D. S. Higginson, K. Salathia.

Coronary Care Uniti, The Ulster, North Down and Ards

Hospitals Trust, The Ulster Hospital, Dundonald.

Recent changes in Mobile Coronary Care (MCC) caused patient anxiety and were described as Bizarre \& Ludicrous in the press. In 1970 MCC was provided by a doctor and nurse team in a dedicated estate car. In 1991 we switched to a dedicated ambulance with a paramedic, doctor and nurse team. In 1993 it became clear that this service was unaffordable. We devised a format to maintain the philosophy of MCC but cheap enough to be supported by management. We report an audit of a taxi driven doctor and nurse system compared with previous systems $(50$ patient samples). Mean delay between patient call and MCC arrival was, 1. using estate car $13^{\prime} 12^{\prime \prime}, 0$ delays $>30^{\prime}, 2$. using ambulance $16^{\prime} 30^{\prime \prime}, 2 \%$ delays $>30^{\prime}, 3$. using taxi $166^{\prime} 36,6 \%$ delays 
$>30^{\prime}$. Longer delays were related to distance travelled. Mean delay between hospital requesting taxi and taxi arrival at hospital ( 330 calls) was $2 ' 34^{\prime \prime}$ with $4.2 \%$ of delays $>6$ '. We believe that MCC must continue, but will need continuing modification to cope with changing challenges.

EARLY DIAGNOSIS OF ACUTE MYOCARDIAL INFARCTION BY A BED-SIDE SEMI-QUANTITATIVE RAPITEX MYOGLOBIN KIT IN ACCIDENT \& EMERGENCY DEPARTMENT

R. Chandler, P. Shah, F. Lavin, K. Daly.

Cardiology Department, University College Hospital, Galway.

Early diagnosis of acute myocardial infarction (MI) will facilitate decision with regard to administration of thrombolysis for maximum myocardial salvage. Bedside diagnostic kits may have advantage over standard laboratory analysis for rapid diagnosis. In a previous trial, we have shown an advantage of myoglobin (Mgb) over other cardiac markers in the early hours of MI. Hence, we compared sensitivity and specificity of bed-side semi-quantitative latex agglutination (Rapitex) kit for $\mathrm{Mgb}$, with that of standard laboratory analysis of creatinine phosphokinase (CPK) in Accident \& Emergency Department (A\&E).

A total of 28 patients ( 20 males \& 8 females), aged less than 75 years (mean of 59.82 yrs), who presented to A\&E with classical history of chest pain with onset less than 12 hours were studied. Venous blood samples were collected for both bed-side Rapitex Mgb kit and serum CPK in A\&E dept. Further blood samples were collected for serum CPK at 12 hours, day 1 and day 2. ECGs were done simultaneously. Of the 16 patients with positive Mgb on admission, 7 had normal CPK ( $<250$ IU/ $)$ and ECG, 4 had normal CPK and abnormal ECG, 2 had elevated CPK and normal ECG and 3 had elevated CPK and abnormal ECG. In the above positive Mgb group, 15 patients had developed ECG changes and high CPK suggestive of MI by day 2. There were 12 patients with negative Mgb on admission; of them, 10 had normal CPK and ECG, 1 had normal CPK and ECG suggestive of acute MI, and the remaining one had elevated CPK with no ECG changes. In this negative Mgb group, only I patient showed evidence of myocardial infarction. Rapitex Mgb showed sensitivity, specificity and positive predictive value of $93.75 \%, 91.66 \%$ and $93.75 \%$ respectively, and CPK showed values of $31.25 \%$, $91.66 \%$ and $83.33 \%$ respectively

In summary, bed-side Rapitex kit for Mgb could be an useful tool for early diagnosis of acute $\mathrm{MI}$ in A\&E department and aid decision making in administration of early thrombolysis

\section{SESSION 2}

PEAK EJECTION FRACTION PRODUCED BY THE ARBUTAMINE PHARMACOLOGICAL STRESS TEST - A COMPARISON WITH CONVENTIONAL BICYCLEEXERCISE

I. C. Steele, A. M. Nugent, S. R. Vallely, N. P. S. Campbell, D. P. Nicholls.

Royal Victoria Hospital, Grosvenor Road, Belfast.

Although most cardiovascular stress tests involve exercise this cannot be performed in all patients. We set out to evaluate a new pharmacological stress testing (PST) agent called arbutamine, a catecholamine with predominantly $B 1$ agonist effects. It was used in conjunction with a computer controlled, closed loop, delivery system. We looked specifically at its effect on left and right ventricular ejection fractions (LVEF and RVEF respectiely). Ten patients with proven coronary artery disease underwent erect incremental bicycle excrcise test (ETT) and supine PST. LVEF and RVEF were measured at rest and at peak stress during both tests, using a high speed, digital multiwire camera and Tantalum 178. Results are shown:

$\begin{array}{lcc} & \text { peak LVEF mean (range) } & \text { peak LVEF mean (range) } \\ \text { ETT } & 51.0(25-70) & 48.5(35-61) \\ \text { PST } & 52.8(36-71) & 52.7(38-78)\end{array}$

There was a significant correlation between the two tests for peak LVEF ( $r=0.72, p=0.02$ ), but no significant correlation for peak RVEF $(r=0.2, p>0.5)$. We conclude that the arbutamine stress test produces similar left ventricular inotropic responses to bicycle exercise despite a change in patient position for the studies), but different right venricular responses

IMPROVED EARLY POSTOPERATIVE CARDIAC FUNCTION ASSOCIATED WITH SYSTEMIC INHIBITION OF FREE RADICAL PRODUCTION

J. G. Coghlan, W. D. Flitter, R. Daly, G. D. Wright, C. D. Ilsley, T. Slate

Harefield Hospital and Brunel University, Middlesex, England.

In this randomised, double blind trial the clinical, haemodynamic and biochemical effects of xanthine oxidase inhibition (XOI) in patients undergoing coronary artery bypass grafting were assessed. Low dose allopurinol pretreatment sígnificantly reduced postoperative inotrope requirement ( $\left.5 / 25 v^{\prime} \mathrm{s} 13 / 25 ; \mathrm{p} 0.01\right)$ and increased the rate of peripheral rewarming $(11,4 \pm 85 \mathrm{hrs} v$ 's $14.4 \pm \mathrm{l}$ hrs; $\mathrm{p} 0.02$ ). Twenty patients (11 placebo) underwent invasive haemodynamic monitoring and intraoperative coronary sinus cannulation. Relative to the placebo group higher cardiac indices were recorded in the active treatment group from four hours postoperatively $(F=8.1 ; D F=1.18 ; p 0.006)$. The net increase in products of lipid peroxidation was significantly less in the active treatment group ( $+94 \pm 40 \mathrm{nmol}$ minutes/g albumin y's $+219 \pm 40 \mathrm{nmol}$ minutes/g albumin; p 0.02). Conclusion. The clinical and haemodynamic benefits of $\mathrm{XOI}$ are preceded by a reduction of intraoperative lipid peroxidation.

RESTENOSIS AFTER SUCCESSFUL PTCA IS INDEPENDENT OF VESSEL SIZE, QUANTITATIVE ANGIOGRAPHIC ANALYSIS IN 3072 PATIENTS

D. P. Foley, R. Melkert, D. Keane, P. W. Serruys.

Thoraxcentre, Etasmus University, Rotterdam, NL

New percutaneous transluminal coronary interventions are reported to be attended by somewhat lower restenosis rates (RR) than balloon angioplasty (PTCA). However, these interventions tend to be restricted to larger coronary vessels. The aim of this study was to objectively evaluate the effect of vessel size (VS) on "restenosis" ( $R$ ), using both a continuous (loss during follow up, \% diameter stenosis at follow up (\% DSfup)) and categorical approach (\% DSfup $\geq 50 \%$ ), in a large homogenous patient group treated by PTCA. Angiography was performed under optimal conditions for quantitative analysis by the CMS system, using multiple projections, matched pre and post PTCA and at 6 months, on 3736 lesions, in 3072 patients, ( $92 \%$ angiographic follow up). Effect of VS on loss and \% DSfup was evaluated by linear regression, and on the occurrence of $\mathbf{R}$, by logistic regression. 
Furthermore, loss, DSfup and RR were compared among lesion deciles ( $D c, n=373$ ) according to VS.

Del Dc2 Dc3 Dc4 De5 Dc6 Dc7 De8 Dc9 Del0 'p

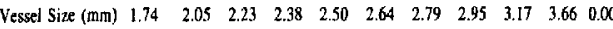
$\begin{array}{lllllllllll}\text { Luminal loss (mm) } 0.29 & 0.28 & 0.27 & 0.28 & 0.32 & 0.29 & 0.29 & 0.35 & 0.34 & 0.28 & 0.47\end{array}$ $\begin{array}{llllllllllll}\text { z DS at fup } \quad 47 \% & 46 \% & 45 \% & 45 \% & 46 \% & 46 \% & 46 \% & 47 \% & 48 \% & 47 \% & 0.64\end{array}$ Restenosis Rate $\quad 31 \% \quad 37 \% \quad 31 \% \quad 34 \% \quad 35 \% \quad 39 \% \quad 37 \% \quad 38 \% \quad 39 \% \quad 36 \%$

In regression analysis, VS did not influence luminal loss ( $p=0.47)$, diameter stenosis at follow up $(p=0.88)$, or occurrence of restenosis ( $p=0.77$ ). Conclusion: by continuous or categorical approach, restenosis does not vary with vessel size. Reported benefits of other devices over PTCA are thus unlikely to be due to less restenosis in larger vessels and may be attributed to intrinsic device specific advantages in provision of larger acute lumen.

\section{IS DECISION ANALYSIS THE BEST WAY TO COMPARE "ACCELERATED" tPA WITH STREPTOKINASE THERAPY?}

J. Kellett.

General Hospital, Nenagh, Co. Tipperary.

A decision analysis was constructed to model the chance events and outcomes likely to occur to a patient with a possible acute myocardial infarction giving aspirin, streptokinase, "accelerated" tPA, or no treatment. The base case was a 60 year old man presenting four hours after the onset of symptoms with a definite acute myocardial infarction, having an anticipated 30 day mortality rate, without aspirin or thrombolytic treatment, of 16 pe cent. Such a patient has a life expectancy at presentation of 10 years and 316 days; treatment with aspirin alone will gain an extra 226 days of quality adjusted life; treatment with aspirin and streptokinase will gain an extra $\mathbf{4 2 0}$ days. Treatment with aspirin and "accelerated" tPA will gain an extra 490 to 514 days, depending on the efficacy of thrombolysis to reduce heart failure assumed. The threshold chance of stroke above which thrombolysis is no longer the preferred treatment is 6.0 percent for streptokinas and 8.3 per cent for "accelerated" tPA. Varying the utility of stroke, and the chance and mortality of major bleed and anaphylaxis throughout their plausible ranges produces only trivial changes in expected outcomes. Provided acute infarction is certain and the chance of dying from infarction is 16 per cent, the cost of

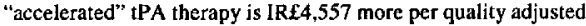
life year saved than treatment with streptokinase

\section{THROMBOSIS FOLLOWING STENTING IN FAILED PTCA: FREQUENCY, CONSEQUENCES, AND PREDICTORS}

\section{J. B. Foley, K. Sridhar, R. I. G. Brown, I. M. Penn.}

Victoria Hospital, London, Ontario, Canada.

One hundred and five Palmaz-Schatz stents were implanted in 60 patients with failed PTCA. Acute stent thrombosis $(<24$ hours) occurred in 3 (5\%) and subacute ( 24 hours-l month) in 7 (11.7\%). Stent thrombosis was associated with $\mathrm{Q}$-wave $\mathrm{MI}$ in 3 and non-Q-wave $\mathrm{MI}$ in 5 . Shown are the frequency of complex morphology (ACC/AHA B2 or C), multiple stent implantation, dissection length $(\mathrm{mm})$, stenosis post stenting and uncovered dissection post-procedure.

$\begin{array}{lccccc} & \text { Comples } & \text { Mallipie } & \text { Dissection } & \text { \% Post } & \text { Uaconred } \\ \text { No Thrombosis }(n=50) & 12 \% & 47 \% & 14 \pm 12 & 25 \pm 13 & 14 \% \\ \text { Thrombosis }(n=10) & 40 \% * & 50 \% & 23 \pm 29 & 40 \pm 25 \# & 60 \% \# \\ \left({ }^{*} p=0.05 \text {, \#p<0.01) }\right. & & & & & \end{array}$

Stent thrombosis was associated with complex lesion morphology, uncovered dissection post-procedure and a greater residual stenosis. An optimum angiographic result is of key importance in reducing stent thrombosis following stenting in failed PTCA.

\section{SESSION 3}

DOES RESTENOSIS AFTER CORONARY INTERVENTION VARY WITH THE DEVICE USED?

D. P. Foley, R. Melkert, V. A. Umans, P. de Jaegere, P. W. Serruys.

Thoraxcentre, Erasmus University, Rotterdam, NL.

Angiographic results 6 months after successful coronary intervention may be evaluated according to the degree of luminal renarrowing (luminal loss) during follow up (fup) or the residua lumen (minimal luminal diameter) at fup (MLDfup). To determine possible anglographic predictors of these results and the influence of the type of device used, multivariate linear regression techniques were applied to quantitative angiographic data ob tained pre and post procedure and at fup in 1560 patients with 1787 lesions, treated by balloon angioplasty, directional atherectomy (DCA), Wallstent (WA) or Wiktor stent (WI) im plantation. Because of the wide range in vessel size (VS), measured luminal changes occurring at intervention and during fup were adjusted accordingly, to derive relative gain ( $R G$, gain/VS) and relative loss (RL, loss/VS). Results: RL was independently determined by RG ( $<<.0001)$ and MLD pre $(p<0.001)$. Furthermore, $R G$ achieved by DCA independently predicted greater $R L$ $(p=0.007)$ and RG by Wallstent, predicted less $R L(p=0.03)$ compared with the other devices. MLDfup was determined by MLD post ( $p<0.0001)$, but also by VS $(p<0.0001)$ and was negatively associated with RG ( $<<0.001)$, but not by the device used. Conclusion: Luminal renarrowing appears predominantly determined by increase at intervention and is influenced by the device used, whereas, residual lumen at follow up depends on vessel size and post-procedural lumen, is negatively associated with relative gain and not influenced by the device. Thus, although the "restenosis process" appears to vary with the device, "late angiographic outcome" does not.

IMMEDIATE AND MEDIUM-TERM RESULTS OF 500 CONSECUTIVE PATIENTS UNDERGOING PERCUTANEOUS TRANSLUMINAL CORONARY ANGIOPLASTY (PTCA) AT A SINGLE INSTITUTION

J. Galvin, M. Codd, A. Hennessy, S. Leavey, E. Keelan, C. McCarthy, D. Sugrue.

Department of Clinical Cardiology, Mater Misericordiae Hospital, Dublin.

Introduction: Although there is an abundance of data on procedural and immediate outcome following percutaneous transluminal coronary angioplasty (PTCA), medium term results have received little attention. We now report clinical follow-up on 500 consecutive patients who underwent PTCA at a single institution between June 1985 and October 1991. 
Results: Mean patient age was 57 years and $71 \%$ were male. $55 \%$ had had unstable angina, $37 \%$ had had a previous myocardial infarction and $5 \%$ had previous coronary bypass grafting (CABG). $81 \%$ of patients had a single vessel dilated, $16 \%$ of patients had 2 vessels dilated and $3 \%$ of patients had 3 vessels dilated. The number of lesions dilated was 1 in $72 \%, 2$ in $19.2 \%, 3$ in $7.2 \%$ and $>3$ in $1.6 \%$. Clinical follow-up was obtained in $99.6 \%$ of patients at 9-94 months (mean 35 months). At follow-up, $48 \%$ of patients had no or rare angina without further intervention. $5.4 \%$ of the original 500 patients had died, $1 \%$ during or within 24 hours of the procedure. $4 \%$ of patients had an MI, $2 \%$ during or within 24 hours of the procedure. $24 \%$ of patients underwent CABG by the time of follow-up, $1.8 \%$ within 24 hours of the procedure. $16 \%$ of patients had a repeat PTCA.

Conclusion: During medium term follow-up after PTCA almost half of the patients had no further symptoms or rare symptoms only without additional intervention. Most of the remaining patients required repeat revascularisation, more often with CABG than with a second PTCA.

\section{DIABETES MELLITUS INCREASES RESTENOSIS FOLLOWING BALLOON ANGIOPLASTY}

D. Keane, D. P, Foley, P. W. Serruys.

Thoraxcenter, Erasmus University, Rotterdam, The Netherlands.

Acute and long-term angiographic outcome of PTCA in 179 lesions in patients with diabetes mellitus (DM) was compared with that of 1891 lesions in patients without $(N)$ diabetes mellitus who participated in multicentre restenosis prevention trials. As vessel size (RVD) was smaller in DM than in N, we therefore examined relative gain (RG) - the acute gain in MLD normalised for vessel size, and relative loss (RL) - the late loss in MLD normalised for vessel size, and net gain index (NGI) - the difference between RG and RL. Percent diameter stenosis (\% DS) in $\mathrm{DM}$ and $\mathrm{N}$ was similar pre and post PTCA but different at followup (fu). The restenosis rate (defined as \% DS>50\%) in N was $30 \%$ and in DM 42\% - rel. risk 1.4 (1.16-1.68).

RVD MLD pre MLD post MLD fu \%DS fu RG RL NGI $\begin{array}{llllllllll}\mathrm{DM} & 2.53 & 0.98 & 1.73 & 1.31 & 50.2 & 0.30 & 0.18 & 0.12\end{array}$

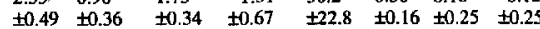
$\begin{array}{llllllllll}\mathrm{N} & 2.61 & 1.02 & 1.77 & 1.50 & 43.8 & 0.29 & 0.11 & 0.19\end{array}$ $\begin{array}{llllllll} \pm 0.53 & \pm 0.39 & \pm 0.35 & \pm 0.57 & \pm 18.6 & \pm 0.16 & \pm 0.21 & \pm 0.22\end{array}$ $\begin{array}{llllllllll}\text { P } & 0.049 & 0.12 & 0.09 & 0.0002 & 0.0004 & 0.64 & 0.0005 & 0.002\end{array}$

Conclusion: Vessel size is smaller in DM than in $\mathrm{N}$ selected for PTCA. Despite an equivalent RG (injury), restanosis following PTCA in DM is greater than in N.

\section{PRIMARY VERSUS SECONDARY STENTING IN FAILED ANGIOPLASTY}

J. B. Foley, K. Sridhar, R. I. G. Brown, I. M. Penn. Victoria Hospital, London, Ontario, Canada.

The safety and efficacy of immediate Palmaz-Schatz stenting (primary stenting) and prolonged inflation with a perfusion balloon (PB) followed by stent implantation if necessary (secondary stenting) for failed PTCA was determined in 46 patients (23 in each group) matched for angina status, vessel and reference vessel diameter. The duration of the maximum inflation achieved in the
PB group was $11 \pm 8 \mathrm{~min}$. Angiographic success ( $<50 \%$ stenosis and TIMI 3 flow) was achieved in $91 \%$ of the primary stent group and $39 \%$ of the $P B$ alone group $(p=0.001)$. Secondary stenting was successful in $8 / 12$ with $P B$ failure. Stent thrombosis occurred in $19 \%$ of all stented patients. Allowing for 1 repeat angioplasty for restenosis $82 \%$ of the stent group and $61 \%$ of the $\mathrm{PB} / \mathrm{second}$ ary stent group were free from angina or major complications at 1 year, $p=0.09$. Primary stenting had a more favourable longterm outcome than PB/secondary-stenting.

\section{BALLOON ANGIOPLASTY OF NATIVE COARCTATION}

B. G. Craig, H. C. Mulholland.

Clark Clinic, Royal Belfast Hospital for Sick Children, Belfast.

Balloon angioplasty has become an accepted form of therapeutic intervention in recoarctation of the aorta following previous surgery. Balloon angioplasty for native coarctation is more controversial and we report our early results with this procedure. Eight procedures were performed in 7 children since January 1991. The mean age was $6.3 \mathrm{yrs}$ (range $1.5 \mathrm{yrs}$ to $14.4 \mathrm{yrs}$ ). The mean peak-to-peak systolic gradient by manometry was $44 \mathrm{msHg}$ (range $36 \mathrm{mmsHg}$ to $56 \mathrm{mmsHg}$ ). This fell to a mean of $21 \mathrm{mmsHg}$ (range $12 \mathrm{mmsHg}$ to $28 \mathrm{mmsHg}$ ) following angioplasty. In one patient the angioplasty was successfully repeated 19 months following the first procedure. There were no major complications. In particular no patient developed an aortic aneurysm or femoral arterial occlusion. Balloon angioplasty provides an alternative to surgery for the relief of native coarctation. Some patients may require repeat procedures for an effective longer term result.

\section{SESSION 4}

DIFFERING PTCA MECHANISMS IN STABLE AND ACUTE CORONARY DISEASE: A STUDY WITH PREAND POST-PTCA INTRACORONARY ULTRASOUND.

P. Kearney, R. Erbel*, L. Koch, J. Ge*, G. Görge*, J. Meyer. Mainz and Essen* University Clinics, Mainz and Essen, Germany.

The wall viewing potential of intracoronary ultrasound (ICUS) has unique advantages in demonstrating the mechanisms of angioplasty. We compared the mechanisms of PTCA in patients (pts) with stable and acute coronary disease (unstable angina and acute myocardial infarction) using pre and post PTCA ICUS.

Of $18 \mathrm{pts}$ included in the study, 9 had stable angina (S) and 9 acute coronary disease (A). 3.5F ICUS catheters attached to a Diasonics console were advanced over the lesion before and after PTCA and images of vessel sites, identified by simultaneously acquired cine recordings, were recorded on video for subsequent offline analysis. Lumen diameter and area, vessel and lesion area and $\%$ stenosis were quantified and morphological changes noted at the same site in the lesion pre and post PICA.

$\begin{array}{lcccc} & \text { Stretch } & \text { Tear } & \text { Dissection } & \text { CLayer (?thrombus) } \\ \text { Stable } & 67 \% & 44 \%, & 22 \% & 0 \% \\ \text { Acute } & 67 \% & 67 \% & 33 \% & 67 \% \\ \chi^{2} \text { p value } & 1.0 & 0.34 & 0.60 & 0.003\end{array}$

A demarcated homogenous inner layer of low echodensity (probable thrombus) was seen only in the pre PTCA images of A pts, which was absent post. A reduction in lesion crosssectional area 
occurred in pts with this layer but not in those without $(p<0.05)$ All other mechanisms and quantitative changes were similar in those with $S$ and $A$

Conclusions. ICUS demonstrates a layer of probable thrombus in the majority of patients with both myocardial infarction and unstable angina. We have shown for the first time a reduction of lesion cross-sectional area after PTCA (consistent with displacement, compression or axial remodelling of thrombus), which is seen only in these patients. Previously described PTCA mechanisms (stretch, tear and dissection) were seen in both $S$ and $A$ pts.

\section{ELECTRICAL WAVEFORM AND ELECTRODE CONFIGURATION IN EPICARDIAL AND} ENDOCARDIAL ATRIAL DEFIBRILLATION

D. Keane, D. Anderson, M. Marrinan, N. Sulke, R. Cooke, G. Jackson, E. Sowton.

Departments of Cardiology and Cardiac Surgery, Guy's Hospital, London, England.

Paroxysmal atrial fibrillation continues to present a therapeutic challenge. The development of an implantable atrial defibrillator would offer significant advantages over current therapeutic approaches. Patient tolerance of atrial shocks, however, would critically depend upon an optimal waveform and electrode configuration.

We first compared the efficiency of a biphasic and monophasic waveform in epicardial atrial defibrillation in 21 patients on cardiopulmonary bypass and then evaluated a right atrial - coronary sinus endocardial electrode configuration using the most efficient of the two waveforms in 16 patients undergoing elective cardioversion of chronic atrial fibrillation and flutter. During epicardial atrial defibrillation, the energy (Joules) required for $80 \%$ success was $3.88(0.59-7.07) \mathrm{J}$ for the monophasic waveform and $0.58(0.41-0.75) \mathrm{J}$ for the biphasic waveform ( $p=0.04)$. Thus, the biphasic waveform was applied during subsequentendocardial defibrillation which was successful in 15 of the 16 patients (4.2 $( \pm 2.9)$ shocks): 8 of 9 patients with chronic atrial fibrillation cardioverted with $6.7( \pm 2.2) \mathrm{J}$ and of the 7 patients with atrial flutter direct conversion to sinus rhythm occurred in $2(0.57$ $( \pm 0.44) J)$, while in 5 the first shock $(0.43( \pm 0.51) \mathrm{J})$ induced atrial fibrillation and subsequent cardioversion required 3.4( \pm 3.3$) \mathrm{J}$

Atrial arrhythmias can be cardioverted efficiently with a biphasic waveform and a right atrial - coronary sinus endocardial electrode system, indicating that an implantable atrial defibrillator would be a feasible treatment modality.

AN ESOTHORACIC PACING SYSTEM FOR ATRIAL, VENTRICULAR AND AV SEQUENTIAL PACING AND ELECTROPHYSIOLOGICAL STUDIES

D. J. McEneaney, J. Anderson, A. A. J. Adgey.

Regional Medical Cardiology Centre, Royal Victoria

Hospital, Grosvenor Road, Belfast BT12 6BA.

A novel esothoracic (ESO) pacing system has been developed capable of atrial (A), ventricular (V) and AV sequential pacing and programmed stimulation. Under light sedation, the flexible polythene gastro oesophageal electrode was swallowed or passed into the stomach. The electrode tip was then positioned in the gastric fundus using an internal pulley system. Four ring electrodes mounted proximally, now lying in the lower oesophagus, were used for $A$ recording and pacing. $V$ pacing used a cathodic point source on the electrode tip and a high impedance chest pad (anode) placed medial to the apex. AV sequential pacing was attempted in 45 patients with stable bradyarmythmias. A capture was achieved in $44 / 45(98 \%)$ subjects and $V$ and $A V$ sequentia capture in 41/45 (91\%), ESO and external (transcutaneous, TC) pacing were compared in 18 patients. $V$ capture was more frequent using ESO pacing (ESO $17 / 18$ (94\%), TC 6/18 (33\%) p $<0.001)$. Threshold current for $\mathrm{V}$ capture was significantly lower with ESO pacing. Emergency ESO V or A pacing was successful in the management of acute bradyarrhythmias ( 3 patients) Electrophysiological studies were performed using ESO and endocardial (ENDO) programmed stimulation. Sinus node re covery time (SNRT) and AV Wenckebach point correlated closely: SNRT $n=12, r=0.92, p<0.001$; AV Wenckebach $n=13, r=0.91$, $\mathrm{p}<0.001$. It was possble to induce and terminate ventricular tachycardia (VT) using ESO programmed stimulation and pacing The rate, morphology and haemodynamics of VT were similar using ESO and ENDO approaches. This ESO pacing system would be useful for emergency pacing. It enables a virtually complete electrophysiological study to be performed withou transvenous catheterisation or access to fluorosscopy.

\section{EPICARDIAL STIMULATION IMPAIRS LOWER OESOPHAGEAL SPHINCTER FUNCTION}

M. T. P. Caldwell, P. Marks, P. J, Byrne, T. N. Walsh, T. P. J. Hennessy, P Crean.

University Dept. of Surgery, St. James's Hospital, Dublin 8.

There is a high incidence of oesophageal disease, particularly gastro-oesophageal reflux disease (GORD) in patients with treated coronary artery disease. The link between these two entities remains unclear. The effect of epicardial stimulation with friction and a nicotene soaked patch (200ug/ml) on lower oesophageal sphincter (LOS) function was studied in 7 adult mongrel dogs. LOS tone was recorded continuously throughout using a sphinctometer.

Results [mean(sem)]

Control 1 Epicardial Control 2 Nicotene friction patch

LOS tone

(sphinctometer) $\quad 13.0(2.2) \quad 6.2(1.7)^{*} \quad 12.8(2.2) \quad 6.9(1.3)^{*}$ Mean blood pressure

$\begin{array}{lllll}(\mathrm{mmHg}) & 92(8) & 72(7)^{*} & 89(7) & 74(5)^{*}\end{array}$

* $\mathrm{p}<0.03$ vs control Wilcoxon signed rank test

These data demonstrate that LOS tone may be reduced by epicardial stimulation and the effect of nicotene suggests that this change may be due to activation of $\mathrm{C}$ afferent vagal receptors in the myocardium of the left ventricle. This may explain the high incidence of GORD in patients with ischaemic heart disease.

\section{SESSION 5}

RELATIONSHIP BETWEEN LIPID PROFILE AND THE PRESENCE AND EXTENT OF CORONARY ARTERY DISEASE

J. Galvin, M. Codd, Leavey, D. Sugrue.

Department of Clinical Cardiology, Mater Misericordiae Hospital, Dublin 7.

Introduction: Serum cholesterol (chol), in particular LDL chol has been shown in numerous cross-sectional and longitudinal studies to be an independent risk factor for coronary artery disease 
(CAD). Apoprotein (apo) B-100, A-I and more recently lipoprotein (a) $[\mathrm{Lp}(\mathrm{a})]$ have also been associated with an increased risk of CAD. It remains unclear however which measurement has the strongest predictive value for the presence or extent of CAD. To assess the relative predictive value of each of these risk factors for the presence or extent of CAD, we measured their levels and assessed cardiovascular risk factors in 157 consecutive subjects undergoing routine diagnostic coronary angiography. All coronary angiograms were read independently and a coronary angiogram scoring system applied.

Results: Elevated apo B-100 ( $\mathrm{p}=0.0001)$, chol $(\mathrm{p}=0.0002)$, $L D L$ chol $(p=0.0005)$, triglycerides $(p=0.0017)$ and $L p(a)$ $(p=0.0337)$ were all significantly associated with the presence of CAD in descending order of significance in a univariate analysis. Total chol alone remained a significant predictor of the presence of $\operatorname{CAD}(p=0.0002)$ with multivariate analysis. Apo B-100 alone was associated with the extent of $C A D$ in both a univariate $(p=0.0103)$ and multivariate analysis $(p=0,05)$.

Conclusions: Although all parameters are significantly associated with the presence of CAD in this population, apo B-100 and total chol possess the strongest association. A longitudinal study is needed to assess the predictive value of each test as a risk factor for the devlopment of atherosclerosis.

EVALUATION OF RIGHT ATRIAL MASS LESIONS USING TRANSOESOPHAGEAL ECHOCARDIOGRAPHY

J. J. Crowley, A. Kenny, P. Dardas, L. M. Shapiro.

Regional Cardiac Unit, Papworth Hospital, Cambridge, England.

The large number of anatomic variants in the right atrium makes differentiation of pathological lesions from normal structures difficult by transthoracic echocardiography (TTE). The improved image resolution of transoesophageal echocardiography (TOE) allows better assessment of the size and characteristics of intracardiac masses. This study investigates the role of TOE in the evaluation of right atrial mass lesions.

TOE was performed in 25 consecutive patients who had an equivocal echogenic structure seen in the right atrium by TTE. Echocardiographic studies were analysed independently by 2 observers without previous knowledge of the clinical data for individual patients.

A definitive diagnosis was made in 23 patients by TOE. Lesions identified included right atrial, thrombus ( 7 patients): right atrial myxoma (3 patients); atrial septal aneurysm (2 patients); Chiari network ( 2 patients); and normal anatomic variant ( 9 patients). Lesion characteristics such as size, morphology and site of attachment to the atrial wall were clearly identified. We conclude that TOE is superior to TTE in the diagnosis of right atrial mass lesions and is useful in guiding appropriate therapy.

PHARMACOLOGY OF A PLATELET GLYCOPROTEIN IIB/IIIA RECEPTOR ANTAGONIST IN PATIENTS WITH STABLE ANGINA PECTORIS

N. Delanty, N. Moran, F. Catella, G. A. FitzGerald, D. J. Fitzgerald.

Centre for Cardiovascular Science, University College Dublin and Mater Hospital, 41, Eccles Street. Dublin 7.

Exposure of the platelet glycoprotein (GP) receptor llb/lla to RGD-containing peptides and other antagonists induces a high affinity state for fibrinogen and the appearance of new epitopes (ligandinduced binding sites or LIBS). Exposure of these sites may mediate platelet interactions with tissue adhesive molecules such as vitronectin, thromboxane (TX) $A_{2}$ generation and platelet aggregation. To explore whether this partial agonist effect altered platelet activity in vivo, we examined the effects of integrelin, a GP 1lb/llla antagonist, in 6 patients with stable angina pectoris. Aspirin was witheld for 10 days prior to study. Integrelin markedly suppressed platelet aggregation to thrombin $(88+5 \%$, mean+SEM) and the thromboxane-mimetic U46619 (93+6\%). In all patients, integrelin increased platelet binding of D3, a monoclonal antibody that identifies a LIBS on the B-subunit. D3 binding correlated strongly with increase in bleeding time for each patient ( $r=0.9$ ). Urinary ll-dehydro-TXB , an index of platelet activation in vivo, was measured in 3 patients, and despite the increased D3 binding, was unaltered by integrelin ( $145 \pm 25$ vs $139 \pm 37 \mathrm{pg} / \mathrm{mg}$ creatinine at baseline). Thus, administration of a GP llb/llla antagonist in vivo results in conformational changes of the receptor, but does not increase platelet activation. Furthermore these conformational changes may be important in mediating the increase in bleeding time seen with these agents.

\section{RESTENOSIS FOLLOWING BALLOON ANGIOPLASTY IS} INDEPENDENT OF GENDER

D. Keane, D. P. Foley, V. Umans, P. W. Serruys.

The Thoraxcenter Erasmus University Rotterdam.

Baseline measurements and acute and long-term results of PTCA, as assessed by quantitative coronary angiography, were compared in 407 lesions in females (F) and 1778 lesions in males (M) who participated in multicentre restenosis prevention trials in Europe \& North America. While vessel size (RVD) and minimal luminal diameter (MLD) pre and post PTCA were slightly smaller in $\mathbf{F}$ than in M, MLD at follow-up (fu) was similar. To permit a direct comparison between vessels of different size, the concepts of relative gain (RG) and relative loss (RL) - the acute gain and late loss in MLD normalised for vessel size, and loss index (Ll) - late loss normalised for acute gain were applied. The restenosis rate ( $\%$ diameter stenosis ( $\% \mathrm{DS})>50 \%$ ) in $\mathrm{F}(27 \%)$ and $\mathrm{M}(31 \%)$ was not significantly different.

$\begin{array}{ccccccccc} & \text { RVD } & \text { MLD pre } & \text { MLD post } & \text { MLD to } & \text { \$DS to } & \text { RG } & \text { RL } & \text { L } \\ \text { F } & 2.53 & 0.97 & 1.69 & 1.44 & 44.5 & 0.29 & 0.10 & 0.32 \\ & \pm 0.51 & \pm 0.39 & \pm 0.37 & \pm 0.60 & \pm 20 & \pm 0.17 & \pm 0.23 & \pm .82 \\ \text { M } & 2.64 & 1.02 & 1.76 & 1.49 & \mathbf{4 5 . 1} & 0.28 & 0.11 & 0.45 \\ & \pm 0.55 & \pm 0.39 & \pm 0.37 & \pm 0.58 & \pm 19 & \pm 0.16 & \pm 0.21 & \pm 4.52 \\ & \mathbf{0 . 0 0 0 1} & 0.02 & 0.0009 & 0.19 & 0.53 & 0.67 & 0.65 & 0.55\end{array}$

$\begin{array}{llllllll}0.0001 & 0.02 & 0.0009 & 0.19 & 0.53 & 0.67 & 0.65 & 0.55\end{array}$ selected for PTCA, their RG and RL are equivalent. Thus acute angiographic outcome and subsequent restenosis following PTCA is independent of gender.

IMPAIRED PULMONARY GAS EXCHANGE IN STABLE CONGESTIVE HEART FAILURE DUE TO ALVEOLAR DYSFUNCTION

D. Moore*, A. Weston, M. Hughes, B. Maurer*, J. Cleland. Cardiac Dept, St Vincent's Hospital, Dublin* and Royal Postgraduate Medical School, London, England.

It has recently been recognised that arterial hypoxaemia may occur during exercise in congestive heart failure, although the mechanisms are uncertain. We have used the Roughton-Forster technique to measure the alveolar-capillary membrane conduct- 
ance for carbon monoxide $(\mathrm{Dm})$, and pulmonary capillary blood volume (VC) during single-breath estimations of diffusing capac ity in 10 male patients with stable congestive heart failure of more than 6 months duration (LV ejection fraction $21.5 \pm 6.8 \%$, age $48 \pm 9 \mathrm{yrs}$ ) on optimum medical therapy with diuretics and ACE inhibitors. Results were compared with 8 sex matched control subjects $(44 \pm 12$ yrs) without evidence of cardiac disease. None of the patients or controls were smokers.

$\begin{array}{lcccc} & \begin{array}{c}\text { DLCo } \\ \text { mumol/min/KPa }\end{array} & \begin{array}{c}\text { DLCO } \\ \text { predicted }\end{array} & \begin{array}{c}\text { Dm } \\ \text { mmolmin/kPa }\end{array} & \begin{array}{c}\text { vc } \\ \text { ml }\end{array} \\ \text { CHF } & 5.69 \pm 1.41 & 57.5 \pm 10.6 & 7.6 \pm 2.6 & 45.1 \pm 21.5 \\ \text { Controls } & 9.80 \pm 2.03 & 96.0 \pm 10.3 & 16.3 \pm 3.8 & 38.0 \pm 12.2 \\ \text { P value } & <0.0001 & <0.0001 & <0.0001 & 0.42\end{array}$

The substantial reduction in $\mathrm{Dm}$ in the patients indicates markedly reduced alveolarcapillary conductance and consequently increased resistance to pulmonary gas exchange. Allowing for increased pulmonary capillary transit time in CHF, this suggests highly abnormal alveolar function in the patient group. Despite severely impaired left ventricular function, $\mathrm{VC}$ was not significantly increased, perhaps as a consequence of intensive diuretic therapy. These data confirm our previous reports of impaired diffusing capacity in congestive heart failure, and suggest that pulmonary gas exchange and hence dyspnoea and functional capacity may be influenced by. alveolar dysfunction in such patients.

\section{PSYCHOLOGICAL IMPACT OF PARTICIPATION IN THE CARDIAC TRANSPLANT GAMES}

H. M. McGee, J. H. Horgan.

Department of Cardiology, Beaumont Hospital, Dublin.

Despite preoperative deconditioning and lowered aerobic capacity for cardiac transplant patients, significant exercise benefits have been documented following hospital-based rehabilitation. Patient-led groups have also developed exercise-based events, e.g. the Cardiac Transplant Games. These may have significant psychosocial, as well as physical, benefits but are also potential sources of stress. This study examined patients from the National Transplant Centre, Mater Hospital, wishing to participate in the 1992 Cardiac Transplant Games in the Netherlands. Twenty patients $(15 \mathrm{~m} / 5 \mathrm{f})$ were assessed to determine medical suitability and prescribe training schedules. Psychological assessment was completed 9 weeks before (T1), the week before (T2) and 8 weeks after the Games (T3). This included physical symptoms, body image, anxiety, mood and health-related quality of life (QoL). One patient was advised not to participate, 5 withdrew ( 3 for health reasons), leaving 14 participants $(13 \mathrm{~m} / 1 \mathrm{f})$, mean age 46.4 (range 31-67) years. Results indicated significant effects for body image and physical symptoms; both improved from T1-T2, this was retained at T3. There was no change in anxiety, mood or health-related QoL over time. Thus participation in a sporting event by well-selected transplant patients was associated with no negative psychological effects but with decreased physical symptomatology and an enhanced body image.

\section{PERCUTANEOUS BALLOON PERICARDIOTOMY IN THE} TREATMENT OF MALIGNANT PERICARDIAL EFFUSION

B. Deb, P. Crean, I. Graham.

Trinity Cardiology, St. James's and Adelaide Hospitals, Dublin 8.

Recurrent pericardial effusion can be manifestation of metastatic malignant disease. Repeat catheter drainage or surgical pericardial window are two common treatment modalities. Percutaneous balloon pericardectomy is an effective way of draining the effusion and preventing recurrence. This is a relatively new technique and is performed under local anaesthetic. We performed this procedure on a female patient diagnosed as having metastatic malignant melanoma who presented with recurrent pericardial effusion with clinical and echocardiographic features of cardiac tamponade. A window was created by causing a tear in the pericardium with a balloon dilating catheter (CRISTAL; $25 \mathrm{~mm}$ diameter, $40 \mathrm{~mm}$ length). The patient tolerated the procedure well and remained asymptomatic from the cardiac point of view over the period of following 4 weeks. This procedure is reasonably safe, performed under local anaesthetic and more importantly obviates the need for surgical procedure in terminally ill patients.

ASSESSMENT OF BODY SURFACE MAPPING IN THE DIAGNOSIS OF ACUTE MYOCARDIAL INFARCTION

C. Cullen, G. Dempsey, G. Wright, L. Martin, G. MacKenzie, J. Anderson, J. Adgey.

Cardiac Unit, Royal Victoria Hospital, Belfast, Northern Ireland.

The additional spatial information provided by body surface maps may improve the early diagnosis of acute myocardial infarction (MI), facilitating the initiation of thrombolytic therapy to 'at risk' patients.

The diagnostic power of a portable ECG mapping device, incorporating an easily applied, screen-printed harness of 64 electrodes, was evaluated. QRS and ST-T iso-integral maps were compared in 83 controls and 61 patients (pts). Controls: 83 (mean age $54 \mathrm{yrs}, 52$ male), with no history of cardiac disease were selected randomly and mapped. Patients: 61 (mean age $64 \mathrm{yrs}, 40$ male) were mapped at first presentation of chest pain suggestive of MI (mean delay from onset of chest pain to map recording was 208 mins). Of these, 36 were mapped outside hospital. Fifty-two (85\%) had definite MI changes on 12-Icad ECG with subsequent confirmatory cardiac enzyme elevation. Nine (15\%) presented with non-diagnostic ECG findings ( $5 / 9$ had $\mathrm{MI}$ confirmed by subsequent significant increase in cardiac enzymes and $4 / 9$ had the diagnosis of angina).

Using the iso-integral measurements to compare MI pts with controls a multiple logistic regression analysis identified $8 \mathrm{sig}$ nificantly predictive sites ( 5 on QRS and 3 on ST-T). The resulting discriminant function (DF) yielded a sensitivity of $90 \%(95 \%$ $\mathrm{CI}=82-99 \%$ ) for the diagnosis of MI with a specificity of $96 \%$ $(95 \% \mathrm{CI}=92-100 \%)$. When the maps of the 9 pts with possible MI were assessed using the DF, 8 were classified as MI, including $4 / 5$ cases subsequently confirmed by significant enzyme elevation.

These results suggest that discriminant function analysis of body surface maps leads to improvement in the earlier diagnosis of $\mathrm{MI}$.

CIGARETTE SMOKING CAUSES INCREASED EXCRETION OF 8-EPI-PROSTAGLANDIN $F_{2 \pi}$, A FREE RADICAL DERIVED PROSTAGLANDIN

N. Delanty, J. A. Lawson, D. I. Fitzgerald, G. A. FitzGerald. Centre for Cardiovascular Science, University College Dublin and Mater Hospital, 41 Eccles Street, Dublin 7

Cigarette smoke contains high concentrations of free radicals. These may be important in the pathogenesis of smoking-related 
diseases, including coronary artery disease, 8Epiprostaglandin $(P G) F_{2 \alpha}$ is formed by free radical peroxidation of arachidonic acid. It has marked biological activity, including platelet activation and vasoconstriction, effects that appear to be mediated through a thromboxane receptor. Formation of this metabolite may be a marker of free radical induced cellular injury. We have documented increased urinary levels of 8-epi-PGF ${ }_{2 \mathrm{c}}$ in paracetamol and paraquot poisoning, conditions that are known to be associated with high levels of oxidative stress. We measured (by gas chromatography-mass spectrometry) urinary 8-epi-PGF in 9 young healthy smokers of greater than 15 cigarettes per day and in 9 young healthy non-smokers. Smokers had a 2.5 fold increase, with a mean ( \pm SD) excretion of $47 \pm 7 \mathrm{pg} / \mu \mathrm{mol}$ creatinine in smokers vs $19 \pm 9$ in non-smokers $(p=0.05)$. Therefore, free radical induced injury occurs in subjects who smoke. 8 -Epi-PGF ${ }_{2 \alpha}$ may play a role in the pathogenesis of unstable coronary syndromes and coronary artery spasm in smokers. As the formation of 8-epi-PGF ${ }_{2 m}$ is not dependent on cyclooxygenase activity, its production would be expected to be unaffected by aspirin therapy.

EFFECTS OF VASODILATOR AGENTS ON

CARDIOVASCULAR HAEMODYNAMICS FOLLOWING INHIBITON OF NITRIC OXIDE SYNTHESIS

N. A. Herity, J. D. Allen, B. Silke, A. A. J. Adgey. Regional Medical Cardiology Centre, Royal Victoria Hospital and Departments of Physiology and Therapeutics and Clinical Pharmacology, Queen's University, Belfast.

Excessive endothelial production of nitric oxide (NO) mediates pathological vasodilatation in septic shock. Pharmacological inhibitors of NO synthesis have recently been used to treat this condition. The aim of this work was to investigate a fall in cardiac output which has been observed to follow this treatment and to test whether it was reversible. Systemic (SVR) and pulmonary (PVR) vascular resistance and cardiac output $(\mathrm{CO})$ were measured in pentobarbitone anaesthetised pigs before and after administration of an inhibitor of NO synthesis - NG-nitro-L-arginine methyl ester (LNAME $10 \mathrm{mg} / \mathrm{kg}$ i.v.) - alone $(\mathrm{n}=8$ ) and followed by nicardipine (NIC $0.05 \cdot 0.2 \mathrm{mg} / \mathrm{kg}$ i.v.: $\mathrm{n}=8$ ), theophylline (THEO $7.5-10 \mathrm{mg} / \mathrm{kg}$ i.4.: $\mathrm{n}=8$ ) or the cyclic GMP (cGMP) selective phosphodiesterase inhibitor M\&B 22948 (M\&B 1 - 5 mg/kg i.v.: $n=8$ ). A control group ( $n=8$ ) received only normal saline.

Results:

Pre-Rx L-NaMe NIC Pre-Rx L-NaMe theo Pre-Rx L-NaME M\&B

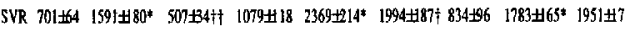

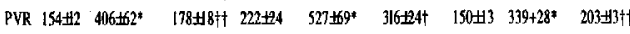

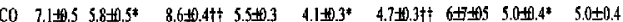
[Mean \pm SEM, SVR and PVR expressed in dyne.sec/ $\mathrm{cm}^{3}$. CO in $1 / \mathrm{min}$. Pre-Rx=Prctreatment value. ${ }^{*} \mathrm{P}<0.01$ vs control group. $\$ \mathrm{P}<0.05$ vs $<\mathrm{L}$-NAME alone. $\dagger \uparrow \mathrm{P}<0.01$ vs L-NAME alone]

Systemic vasoconstriction following L-NAME was completely reversed by nicardipine and partly reversed by theophylline with complete and partial restoration of CO respectively. M\&B did not affect either variable possibly due to total inhibition of cGMP synthesis by L-NAME. All three agents partly reversed pulmonary vasoconstriction. The results suggest that the observed fall in cardiac output (L-NAME) is caused by increased afterload on the heart and is reversed by nicardipine and less potently by theophylline. These findings may prove valuable to those using inhibitors of NO synthesis to treat septic shock.
EFFECTS OF CANDOXATRIL, A NOVEL ENDOPEPTIDASE INHIBITOR, COMPARED TO FRUSEMIDE IN HEART FAILURE

D. B. Northridge, N. C. Jackson, M. J. Metcalfe, H. J. Dargie.

Western Infirmary, Glasgow and Pfizer Central Research, Sandwich.

Atrial natriuretic factor (ANF) has diuretic and vasodilator effects in chronic heart failure (CHF). ANF is degraded by neutral endopeptidase (NEP), and inhibitors of this enzyme may potentiate its beneficial effects in CHF. In this double-blind, randomised trial candoxatril (C), a NEP inhibitor, was compared to frusemide (F) in patients with mild CHF. Thirty male patients with lef ventricular dysfunction (echocardiographic fractional shortening $<25 \%$ ) and reduced exercise capacity were randomised to $C$ $200 \mathrm{mg}$ bid, C $400 \mathrm{mg}$ bid or F $20 \mathrm{mg}$ bid for 9 days of continuous therapy. The initial diuresis and 24 hour sodium excretion following both doses of $\mathbf{C}$ was similar to $\mathbf{F}$. Resting ANF levels increased 2- to 3-fold four hours following C $200 \mathrm{mg}$ and $\mathrm{C}$ $400 \mathrm{mg}$, and remained significantly elevated after 9 days. Conversely, ANF levels fell by $50 \%$ following $\mathrm{F}$. There was no rise in plasma renin (PRA) with C $200 \mathrm{mg}$ bid and C $400 \mathrm{mg}$ bid, compared with a 4-fold increase after $F$. At peak exercise, ANF levels were significantly higher and PRA significantly lower after $C$ compared to $F$. Despite a comparable diuresis and natriuresis candoxatril did not stimulate renin secretion, either at rest or on exercise, in marked contrast to frusemide. This novel diuretic merits further study in heart failure.

\section{MAGNETIC RESONANCE IMAGING PLANES FOR THE} THREE-DIMENSIONAL ANATOMICAL

CHARACTERISATION OF HUMAN CORONARY ARTERIES

J. J. Crowley, A. R. C. Gates, C. L-H. Huang, A Gresham, T. A. Carpenter, L. D. Hall, L. M. Shapiro.

Regional Cardiac Unit, Papworth Hospital and Dept. of Medicinal Chemistry, University of Cambridge, England.

Magnetic resonance imaging (MRI) has the potential to provide a noninvasive means of obtaining 3 dimensional (3-D) images of coronary arteries. However, because of difficulty visualising vessels of such small size and mobility, images of only the proximal parts of the coronary arteries have been obtained in vivo. This study investigates a stratagy to allow the 3-D reconstruction of the coronary arteries in vitro.

Seven cadaveric human hearts were pressure perfused with formalin to maintain coronary artery patency. A series of tomographic planes were devised based on the longitudinal and transverse axis of the heart which allowed imaging of the main coronary arteries in cross-section and longitudinally. These images were then reconstructed into a 3-D image using commercial software. Using this imaging protocol 3-D reconstructions of the main coronary arteries were possible in all 7 cadaveric specimens. The left main, left anterior descending, left circumflex and right coronary artery were visualised for mean lengths of $10 \mathrm{~mm}$ (range 6-13), $24 \mathrm{~mm}$ (range 2.0-3.5),30 $\mathrm{mm}$ (range 13-38) and $55 \mathrm{~mm}$ (range $31-82$ ) respectively.

This study has developed an imaging protocol which allows 3-D reconstruction of the main coronary arteries in vitro. Such an approach may be potentially useful for the systematic clinical imaging of human coronary arteries in vivo. 
DETECTION OF SHOCKABLE VENTRICULAR TACHYCARDIA BY IMPEDANCE CARDIOGRAPHY USING TWO ECG/DEFIBRILLATOR PADS

P. W. Johnston, J. D. Allen, G. Dempsey, J. Jossinet, Z. Imam, J. Anderson, A. A. J. Adgey.

Cardiac Unit, Royal Victoria Hospital, Belfast.

Ventricular tachycardia (VT) may present with insignifican or significant (requiring DC shock) haemodynamic disturbance. To distinguish between them automatic external defibrillators use heart rate alone which is insufficient. The impedance cardiogram (ICG), as a non-invasive haemodynamic indicator, may improve the accuracy in detecting shockable VT. This study compared heart rate $(>200 / \mathrm{min})$ with the ICG recorded by a novel electrode configuration, using $2 \mathrm{ECG} /$ defibrillator pads attached to the anterior chest wall between the $3 \mathrm{rd}$ and 7 th intercostal spaces. In 8 anaesthetised pigs the ICG and cardiac output $(\mathrm{CO})$, measured by thermodilution were recorded simultaneously, firstly during ventricular pacing and then during VT. During pacing at 180,240 and $300 / \mathrm{min}$ and following VT induced with ouabain, the peak ICG $(\mathrm{dz} / \mathrm{dt}(\max ))$ was averaged over 10 beats and the mean $\mathrm{d} z$ dt(max) correlated with $\mathrm{CO}$ by thermodilution. For the pacing and VT studies the correlation coefficients were $r=0.40$ $(y=0.036 x+0.159, p<0.001, n=174)$ and $r=0.54(y=0.051 x+0.079$, $\mathrm{p}<0.001, \mathrm{n}=127$ ) respectively. During VT, $\mathrm{CO}<3 \mathrm{~L} / \mathrm{min}$ was considered shockable. Using the regression equation from the VT study $\mathrm{CO}<3 \mathrm{~L} / \mathrm{min}$ correlated with $\mathrm{dz} / \mathrm{dt}(\max )<0.23 \Omega / \mathrm{s}$. Using heart rate alone ( $>200 / \mathrm{min}$ ) shockable VT was detected with $29 \%$ (6/21) sensitivity and $28 \%(16 / 58)$ specificity and with dz $\mathrm{dt}(\max )<0.23 \Omega / s$ alone, sensitivity was $62 \%(13 / 21)(\mathrm{p}<0.05)$ and specificity $83 \%(48 / 58)(p<0.01)$. Thus the ICG recorded by a novel electrode configuration was superior to heart rate in determining shockable VT.

\section{A SIMPLIFIED APPROACH TO ABLATION OF THE \\ SLOW PATHWAY LIMB OF ATRIO-VENTRICULAR NODAL REENTRANT TACHYCARDIA}

\author{
R. Sheahan, D. Newman, P. Dorian.
}

Division of Cardiology, St. Michael's Hospital, Toronto, Ontario, Canada.

Selective ablation of the slow pathway with radiofrequency (RF) energy is an effective therapy of atrio-ventricular noda reentrant tachycardia (AVNRT). Two methods can be used to achieve this result; an anatomical or an electrical guided approach. We hypothesised that a simplified approach combining anatomic and electrogram characteristics would be safe and effective. This approach involved RF energy application to the inferior aspect of Koch's triangle. inferior and posterior to the His bundle deflection. At this site an A:V signal ratio of $1: 3$ was sought with fractionation of the A electrogram. A deliberate attempt not to selectively record slow pathway potentials was made. Absence of inducible tachycardia and/or echo beats were used as markers of procedural success. Sixteen consecutive patients were studied off drugs. Fifteen were female with a mean age of $52.8 \pm 4.2$ years. Overall success was achieved in $15(94 \%)$ patients. Two patients had symptom recurrence; both underwent repeat ablation (1 success, 1 failure). Two patients who had an initial failed ablation were successfully ablated at a later attempt. The mean ablation procedure time was 46.2 - $11.8 \mathrm{~min}$. in duration. The RF applications were delivered on a mean of 7.7 \pm 2.8 times per procedure. Junctional tachycardia occurred in 7
$(35 \%)$ cases and six of whom had a successful outcome. None of the patients developed transient or permanent heart block or other complications. At follow up 2 months later all patients were asymptomatic and off all medications.

In conclusion, a simplified approach to slow pathway ablation achieves a high success rate with few complications. It does no require a search for discrete potentials or extensive ablations often required with a strictly anatomical approach

CARDIOVASCULAR MORTALITY AND SOCIAL CLASS: EIGHT YEARS OF DATA FROM A CARDIAC REGISTER

R. Meleady, K. S. Tan, C. O'Brien, I. H. Graham Adelaide Hospital, Dublin 8.

There is an inverse relationship between social class and cardiovascular mortality. The mechanisn of this relationship is obscure. Using data from the Meath-Adelaide Cardiac Resiste collected between 1985 and 1992, the best index of social class that showed this relationship was determined. The factors that may mediate this relationship were examined

Indices of social class examined were: medical card eligibility occupation, education attainment levels and employment status. Of these, medical card eligibility was the best index of the inverse relationship between social class and mortality.

Univariate analysis suggests that card holders were older. more likely to be female, to smoke and to have a complicated myocardial infarction. They are less likely to have a positive family history (table).

$\begin{array}{lll} & (\mathrm{n}=640) & (\mathrm{n}=436) \\ \text { In-hospital mortality \% } & 9.7 & 21.7 \\ \text { Mean age (SD) } & 60(11) & 70(10)^{*} \\ \text { Female \% } & 30 & 49^{*} \\ \text { Positive family history \% } & 53 & 40 \\ \text { History of hypcrtension \% } & 29 & 32 \\ \text { Serum cholesterol mmol// (SD) } & 6.2(1.3) & 5.9(1.4) \\ \text { Smokers \% } & 78 & 65 \\ \text { Complicated myocardial infarction \% } & 37 & 47 \\ \text { Infarct topography: } & & \\ \text { Anterior septal/lateral infaret \% } & 46 & 48 \\ \text { Inferior/posterior infarct \% } & 41 & 41\end{array}$

$*=p<0.01$

However, multiple logistic regression analysis showed that the relationship between medical card eligibility and cardiovascular mortality was independent of the variables above. The relationship between social class and mortality do not appear to be mediated by the major cardiovascular risk factors and infarct site.

MULTISITE INHIBITION OF THE PLATELET

GLYCOPROTEIN IIb/IIIa RESULTS IN GREATER

SUPPRESSION OF PLATELET ADHESION TO

FIBRINOGEN

P. Maderna, N. Moran, D. Fitzgerald.

Centre for Cardiovascular Science, Mater Hospital and U.C.D., Dublin.

Platelet aggregation is mediated by the interaction between platelet glycoprotein (GP) IIb/IIla and an Arg-GlyAsp (RGD) sequence in fibrinogen. Compounds that mimic this sequence prevent platelet aggregation and are being assessed as antiplatelet therapy. However, other sites on GPIIb/IIIa may also be involved. 
Fibrinogen induces the expression of a novel epitope on the receptor that is recognised by a monoclonal antibody, D3. Blockade of this site does not inhibit platelet aggregation, but abolishes clot retraction, a platelet-mediated response. We addressed the hypothesis that inhibition of multiple sites would be more effective in preventing the platelet-fibrinogen adhesion by examining the interaction between anti-D3 and RO43-5054 and RO44-9883 two peptidomimetic antagonists of the RGD binding site. Adhesion was measured by incubation of washed human platelets to fibrinogen coated on microtitre plates and by scanning electron microscopy. Preincubation of platelets with RO-43 or RO-44 4.8 uM induced a $31 \pm 8 \%(n=6)$ and $44 \pm 6 \%(n=6)$ reduction in platelet binding. In contrast, anti-D3 $0.3 \mu \mathrm{g} / 2 \times 10^{7}$ cells increased binding by $59 \pm 4 \%(n=3)$, suggesting that anti-D3 induced an active conformation of the receptor. The combination of anti-D3 and the RO compounds markedly inhibited binding of platelets $(76 \pm 5 \%$, $n=3$ for RO43 and $84 \pm 5 \%, n=3$ for RO-44). In conclusion, the GPIIb/IIIa exhibits multiple recognition sites for fibrinogen. Intibition of one site alone may result in incomplete inhibition of platelet activity in vivo.

\section{CARDIOPROTECTIVE LIPPOPROTEIN ALTERATION} RESULTING FROM CONSUMPTION OF

\section{MONOUNSATURATE RICH BUTTER PRODUCED BY A MODIFIED BOVINE DIET}

D. M. O'Callaghan, S. M. Rafferty, M. C. Canton, B. F. Connolly, J. H. Horgan.

Department of Cardiology, Beaumont Hospital, Dublin 9.

Intake of increased monounsaturated (MFA) and polyunsaturated (PFA) fatty acids reduce LDL cholesterol but PFA may also reduce HDL cholesterol. By modifying the bovine diet a butter and cheese rich in MFA was produced. We compared the effects of dietary inclusion of MFA rich butter and cheese (diet B) to regular butter and cheese (diet A) and PFA rich butter and cheese (diet $C$ ) in 30 patients, ( 1 excluded) 23 male (mean age of 56.4 years) with type IIa hyperlipidaemia complying well to an AHA step 1 type diet. Using a double change over design and latin square, patients were randomised to diets A, B and C for 6 weeks each. The effects of these diets on serum cholesterol, triglycerides, HDL, LDL, Cholesterol/HDL ratio. HDL2, HDL3 and LPa were observed. Otherwise diets and medications were unchanged as observed by full dietary analysis. Adjusted means and standaro errors were estimated using a linear model and a two tailed $\mathrm{T}$ test was used to compare means. MFA rich butter and cheese resulted in a significant elevation in HDL3 (0.98 to $1.11 \mathrm{mmol} . \mathrm{LP}<0.05$ ) compared to baseline. Period $C$ resulted in significant reductions in total HDL compared to period A and B $(1.28$ to $1.22 \mathrm{P}<0.05$ and 1.31 and $1.22 \mathrm{P}<0.05$ ). Otherwise no significant changes were observed in serum lipids. This study suggests that inclusion of butter/cheese rich in monounsaturates to the diet of mildly hyperlipidaemic patients results in beneficial serum lipoprotein changes.
INADEOUATE MANAGEMENT OF HYPERLIPIDAEMIA FOLLOWING CORONARY BYPASS SURGERY

D. B. Northridge, M. B. Buchalter, A. Shandall, A. Rees.

Departments of Cardiology, General Medicine and Medical Audit, University Hospital of Wales, Cardiff.

Lipid lowering therapy is well established in secondary prevention, therefore a retrospective audit of the detection and management of hyperlipidaemia in 100 consecutive $C A B G$ patients was carried out (mean age 61, range 44-78). No recorded lipid measurement was present in 17 cases, in a further 5 cases lipid levels had been measured but the results were not in the notes. The median (range) total cholesterol was $6.7 \mathrm{mmol} / / \mathrm{l}(4.0$ $11.7)$ and the median triglyceride was $2.1 \mathrm{mmol} / \mathrm{l}(0.6-18.4)$. These results were included in the discharge summary in 37 cases $(45 \%)$. Of the 41 patients with a serum cholesterol above the median, only $12(27 \%)$ were referred to a dietician, and $8(19 \%)$ were prescribed lipid lowering drugs. These interventions were no more frequent than in patients with cholesterol levels below the median [ $18(42 \%)$ referred to dietician and $4(10 \%)$ on drugs] Of 16 patients with total cholesterol $>8 \mathrm{mmol} / \mathrm{l}$, only 5 were referred to a dietician and 4 were prescribed lipid lowering drugs.

Although $83 \%$ of CABG patients had their lipids measured only the minority with a high cholesterol were managed appropriately. As a result of this audit local guidelines for the management of hyperlipidaemia in CABG patients have been implemented.

COMPARISON OF IONIC AND NON-IONIC DYES IN CORONARY ANGIOGRAPHY: AN ASSESSMENT OF SAFETY AND COST

B. Deb, L. Rajan, R. Sheehan, N. Ghaisas, P. O'Callaghan, P. Crean, M. Walsh, G. Geraty.

Trinity Cardiology, St. James's Hospital, Dublin.

In a randomised, double-blind study we compared the safety and cost of ionic (Urografin) vs, non-ionic (Niopam) contrast media in a select low risk group of patients undergoing routine daycase coronary angiography. The enrolment criteria for this study were patients who could perform an exercise test for at leas 6 minutes. Persons with known allergies, valvular heart disease poor left ventricular function and/or asthma were excluded from the study. $105 / 295(35.5 \%)$ patients were enrolled between March and July 1993. 24/55 (44.1\%) patients in the Urografin group had transient minor symptoms during the procedure compared to 25/50 (50\%) in the Niopam group (p-NS). 18/55 (32.3\%) patients in the Urografin group had transient cardiovascular events i.e. hypotension, bradycardia, PVC's and T wave changes during the procedure compared to $9 / 50(18.7 \%)$ in the Niopam group ( $p-N S)$. None of the patients in either group had any serious adverse events or required any intervention medication. Niopam costs 6 times more than Urografin ( $57.30 \mathrm{vs.} 19.50$ for $100 \mathrm{mls}$ ) We concluded that Urografin can be safely used as a contrast media in a select low risk group of patients undergoing routine coronary angiography at a lower cost compared to Niopam. 